\title{
LHM Filter for Removal Salt and Pepper with Random Noise in Images
}

\author{
Vishnu Prasad Jhariya \\ M.Tech (Final Year) \\ CSE (SISTec), Bhopal (M.P.)India
}

\author{
Ram Gopal Kashyap \\ Assistant Professor \\ CSE (SISTec), Bhopal (M.P.), India
}

\begin{abstract}
In today's modern world, the image quality will be as good as the more information we can get from that image as an image of a planet comes to us, we will clean the image quality as ours about the Planet, But the more information will be able to achieve the integration of mixed noise in the image, we cannot get more information A LHM filter is proposed to remove high density salt $\&$ pepper noise from digital images and outer range noise. The proposed filter works in two stages, in the first stage the noisy pixels are detected and in the second stage each noisy pixel is replaced by the mean value of noise free pixel of $3 \times 3$ the performance of proposed filter is compared with the existing "Impulse Noise Removal Using MDBUTMF with Histogram Estimation filters “, The proposed filter shows better performance as compared to the above mentioned filters for noise removal from different gray scale images.
\end{abstract}

\section{Keywords}

Image de-noising, Image classification, Image restoration and decomposition, Impulsive Noise, MDBUTMF.

\section{INTRODUCTION}

The increase in the digital world, the role of digital images is becoming very much a gift, do not let the man in the coming future in which Planet Make my perch, and it is even possible, today our spacecraft reached the planet and If the incoming image is more clear if we could get more information about the planet Mars, and life and increases the chances. We propose an image filter to remove salt and pepper noise in image with random noise in the image. The color is a spot or mark which comes from the color of his neighborhood does not match, it removes him, namely the filter to the middle value in the neighborhood of pixel values is checked as well as the filter examines the flow of color If the center pixel value, then it would have followed a flow color the filter assumes the noise-free, but the center pixel value does not follow any direction, so that the filter does wrong center pixel value, and the filter of the NOISY pixel value is detected and corrected.

\section{PROBLEM STATEMENT}

"Impulse Noise Removal Using MDBUTMF with Histogram Estimation "[1] this filters checks only salt and pepper noise. He has even pixel value is in the range of 0-4 and 251-255, all of them agree that the noise is filtered. But it is also possible that the color of the image is in the same range or a line in the image to be in the same range or maybe that might be changing the color of the image. This filter checks only value. It is also possible that all the neighboring pixels in a $3 \times 3$ window are a different color from the center pixel color this filter not check for this problem and not completely remove the noise.

\section{OBJECTIVE OF WORK}

Our goal is that the image that came to salt and pepper noise is completely removed. And also check the random noise pixel value but before that, black or white color in the image to check it's exactly what the color is not a color image. After that delete salt $\&$ pepper noise of the image and give the attractive and smooth look into the image. Could we get more information from the image?

\section{PREVIOUS WORK}

\subsection{Impulse Noise Removal Using MDBUTMF with Histogram Estimation}

Many image filters have been discovered yet, but not completely remove the salt and pepper noise in images with random noise in the image. In the same category in 2015, which was a salt \& Pepper Noise Removal Filter "Impulse Noise Removal Using MDBUTMF with Histogram Estimation "[1] has been named to the filter does not delete it completely salt and pepper noise. And the one more image de-noising filter named "A Modified Nonlinear Filtering Technique for Removal of High Density Salt and Pepper Noise" This filter also removes the salt and pepper noise in image. But the weak point of this filter does not remove the random noise in image and the working of this filter for firstly, the Unsymmetric Trimmed algorithms will be applied on input images and after the de-noising image again passed to the given filter the select the mask window with the center of $\mathrm{Yi}, \mathrm{j}$ if the center pixel value is less than 25 than this filter will show uncorrupted pixel value but the center pixel value is greater than 25 this filter examine the center pixel value is corrupted and again check if the center pixel value is greater than 127 if the center pixel value is less than 127 than this filter examine the center pixel value is corrupted and center pixel value is replace by the zero and if the center pixel value is greater than 127 than this filter examine the center pixel value is corrupted and the center pixe value is replace by the 255 the weak point of this filter is that it is only implement for black and white image, another weak point of this filter is that it does not detected the random noise in image and the black pixel value of white image is zero [2].The flow chart of "Impulse Noise Removal Using MDBUTMF with Histogram Estimation is given below 


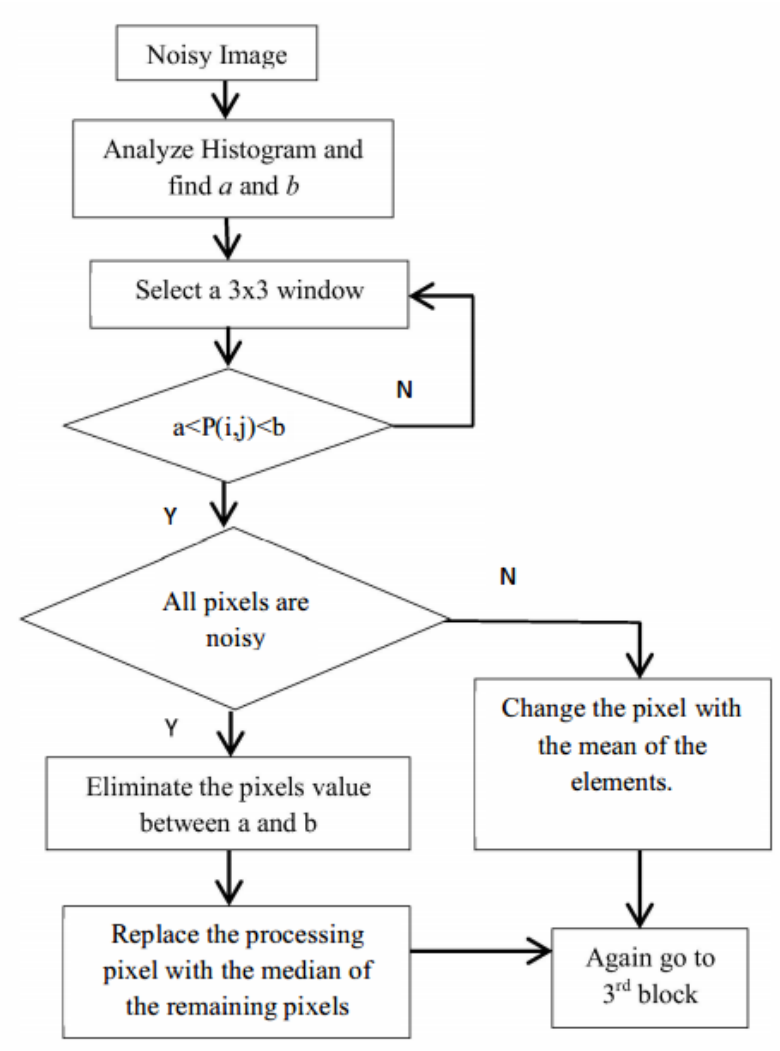

Figure 4.1 MDBUTMF with Histogram Estimation [1]

This filter which together with salt and pepper noise is not it removes salt \& Pepper noise and the one more week point of this filter to consider as the pixel value from 6 to 250 is corrected and outside the value is noise.

It is also possible that the image on the color white and some black, some places do. This filter removes all the noise should be treated.

\subsection{A Modified Nonlinear Filtering Technique for Removal of High Density Salt and Pepper Noise}

This filter also removes the salt and pepper noise in image. But the weak point of this filter does not remove the random noise in image and the working of this filter for firstly, the Unsymmetric Trimmed algorithms will be applied on input images and after the de-noising image again passed to the given filter the select the mask window with the center of $Y_{i, j}$ if the center pixel value is less than 25 than this filter will show uncorrupted pixel value but the center pixel value is greater than 25 this filter examine the center pixel value is corrupted and again check if the center pixel value is greater than 127 if the center pixel value is less than 127 than this filter examine the center pixel value is corrupted and center pixel value is replace by the zero and if the center pixel value is greater than 127 than this filter examine the center pixel value is corrupted and the center pixel value is replace by the 255 the weak point of this filter is that it is only implement for black and white image, another weak point of this filter is that it does not detected the random noise in image and the black pixel value of white image is zero or 255 the flow chart of "Modified Nonlinear Filtering Technique for Removal of High Density Salt and Pepper Noise" is given below[2]

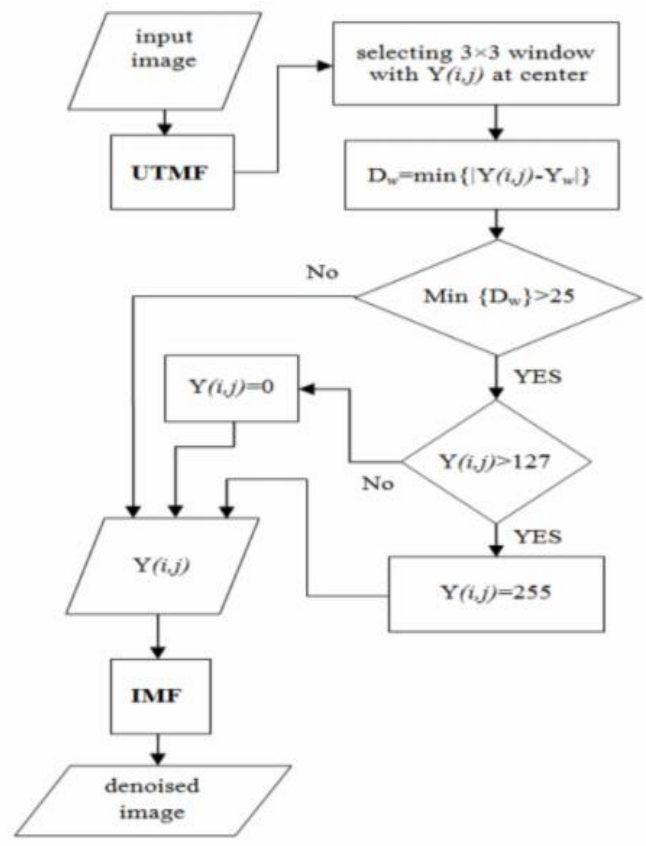

Figure 4.2 The flow chart of "Modified Nonlinear Filtering Technique for Removal of High Density Salt and Pepper Noise"

\section{PROPOSED METHODOLOGY}

In the proposed filter LHM Filter the average value of noise free pixel in selected window is calculated and noisy pixels are replaced by average value of noise free pixels in selected window. The proposed filter is divided in two stages, first stage to Indentify noisy pixels and second stage to remove noisy pixels. These two stages are described in the following subsections.

\section{Suppose the corrupt image is below:-}

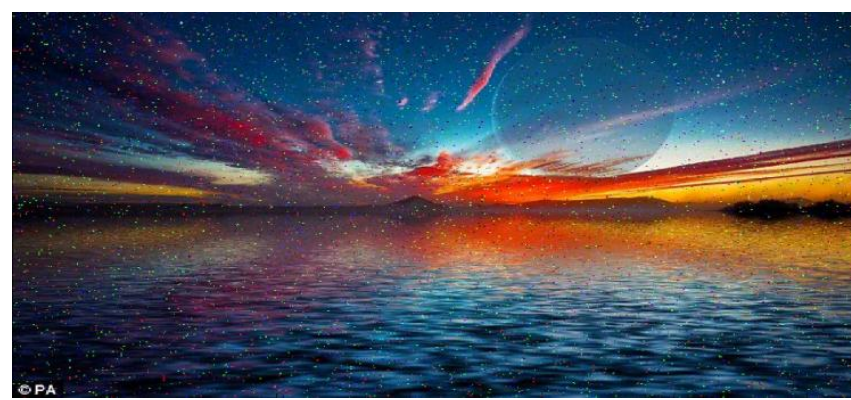

Figure 5.1 Corrupt image

Noise Detection In this stage the main purpose is to identify the "noisy Pixel" and "noise free pixels". It is described as follows:

Let $\mathrm{X}_{\mathrm{ij}}$ denotes the image corrupted by noise. For each pixel $\mathrm{X}_{\mathrm{ij}}$ a 2-D sliding window of size $3 \times 3$ is selected in such a way that the current pixel lies at the centre of the sliding window.

The proposed algorithm first detects the noisy pixel. If the current processing pixel lies inside the dynamic range [0 255] and the value of current pixel are between the left pixel value and the right pixel and also up pixel value and the down pixel value then it is considered as a noise-free pixel. Otherwise it is considered as a noisy pixel and replaced by a value using the proposed algorithm. 
Step 1. Insert the Noisy image.

Step 2. A 2-D window $\mathrm{W}_{3 \times 3}$ of size $3 \times 3$ is selected. Assume that current pixel under processing is $X_{\mathrm{ij}}$.

Step 3. Check the pixel value under 0 to 255 if yes then go to Step 4 otherwise go to Steps 5.

Step 4. Checks the random noises if yes then go to Step 5 otherwise go to Steps 8 .

Step 5. Select the corrupted pixel of $\mathrm{W} 3 \times 3$ and Select the corrupted neighborhood pixel value

Step 6. Calculate the average uncorrupted neighborhood pixel value

Steps 7. Replace the noisy pixel value by the average pixel value and go to Step 3 .

Steps 8 Restore image pixel value and go to Step 3

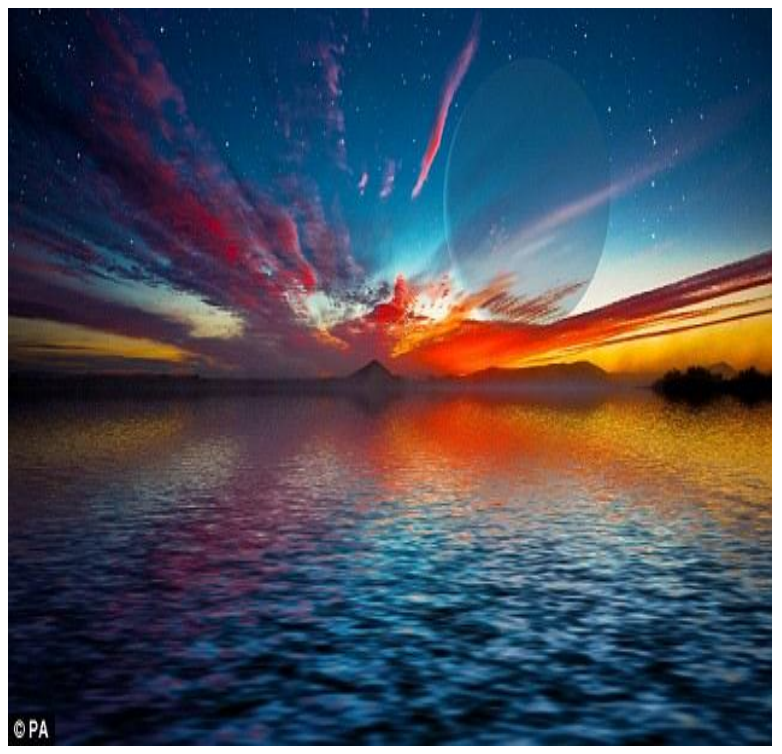

Figure 5.2 Filtered image

Compare real picture, corrupt picture, and filtered picture

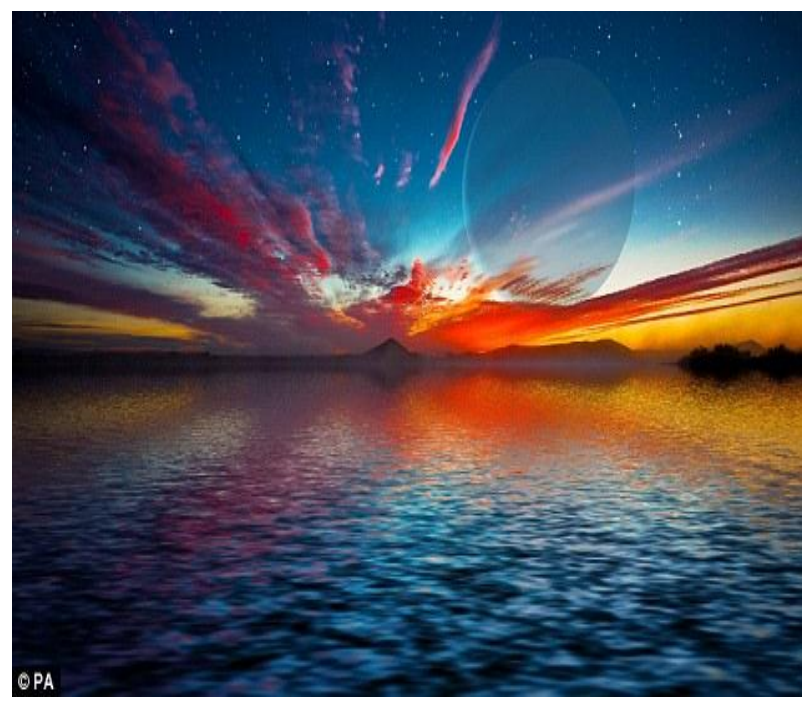

(a)

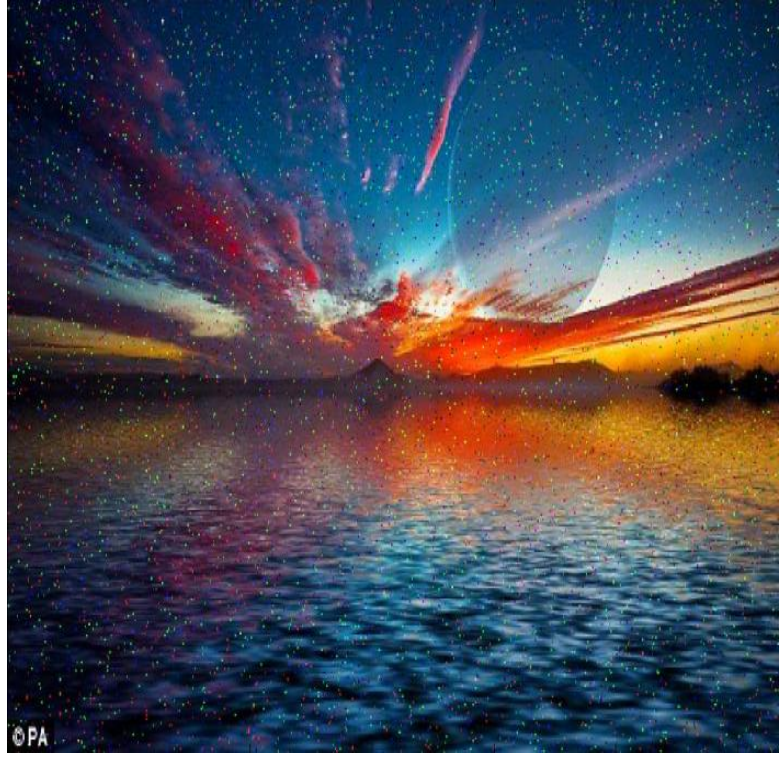

(b)

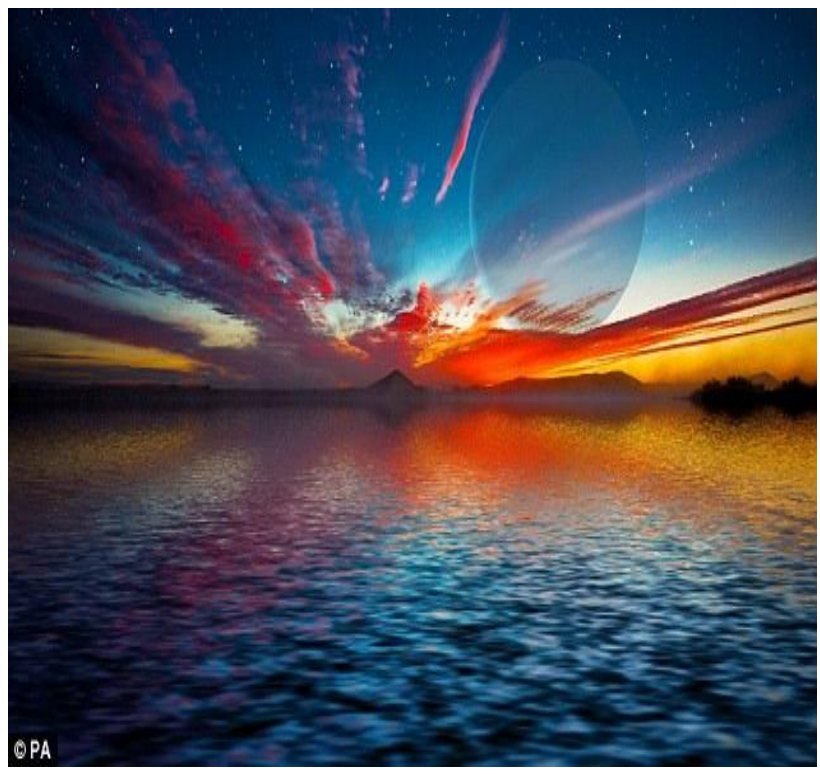

(c)

Figure 5.3 (a) Real image, (b) Noisy image, (c) Filtered image 
The flow chart of propose algorithm is given below:-

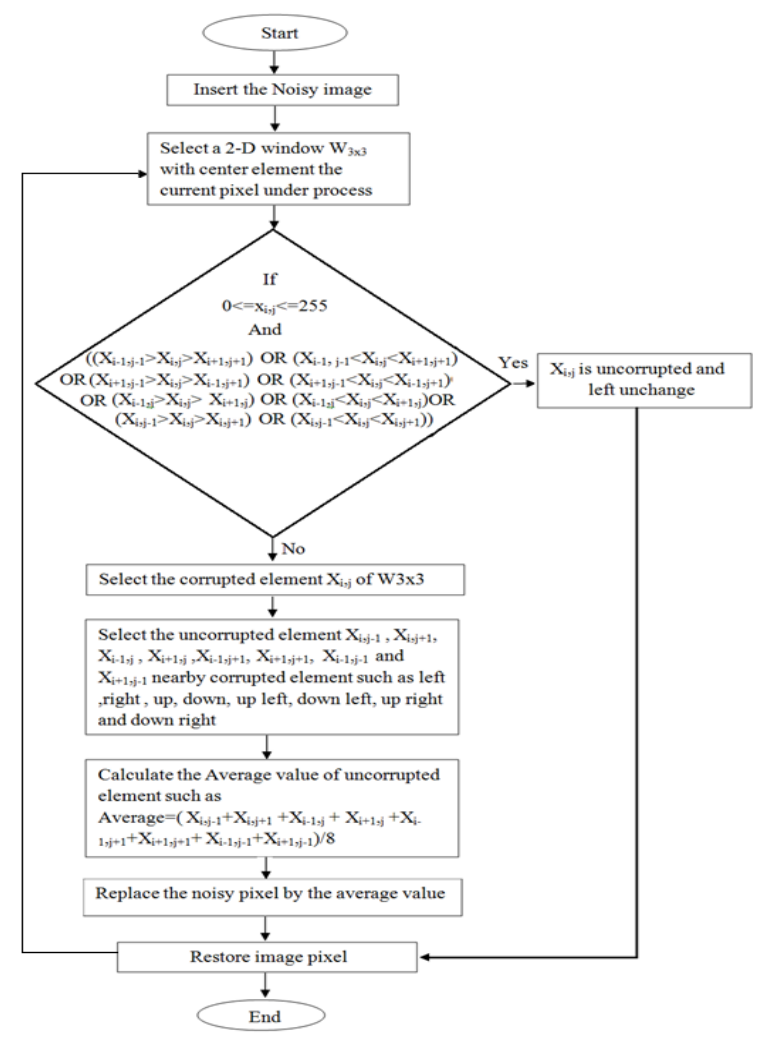

Figure 5.4 Flow chart of LHM Filter

\section{EXPLANATION}

1 Suppose the above program find the error in imageAccording to the following table

Table 6.1. Show how determine the error

\begin{tabular}{|c|c|c|}
\hline $\mathbf{X}_{(\mathrm{i}-1),(\mathrm{j}-1)}$ & $\mathbf{X}_{(\mathrm{i}-1),(\mathrm{j})}$ & $\mathbf{X}_{(\mathrm{i}-1),(\mathrm{j}+1)}$ \\
\hline $\mathbf{X}_{(\mathrm{i}),(\mathrm{j}-1)}$ & $\mathbf{X}_{\mathrm{i}, \mathrm{j}}$ & $\mathbf{X}_{(\mathrm{i}),(\mathrm{j}+1)}$ \\
\hline $\mathbf{X}_{(\mathrm{i}+1),(\mathrm{j}-1)}$ & $\mathbf{X}_{(\mathrm{i}+1),(\mathrm{j})}$ & $\mathbf{X}_{(\mathrm{i}+1),(\mathrm{j}+1)}$ \\
\hline
\end{tabular}

2. If the color of way in top to bottom than the image are not corrupt and the color of flow are given below table

Table 6.2 table show flow of color from top to bottom

\begin{tabular}{|l|l|l|}
\hline 17 & 14 & 85 \\
\hline 77 & 12 & 25 \\
\hline 23 & 11 & 24 \\
\hline
\end{tabular}

3 Else if the color of way in bottom to top than the image is not corrupt and the color of flow are given below table

Table 6.3 table show flow of color from bottom to top

\begin{tabular}{|c|c|c|}
\hline 90 & 145 & 144 \\
\hline 10 & 210 & 11 \\
\hline 245 & 100 & 200 \\
\hline
\end{tabular}

4 Else if the color of way in left to right than the image is notcorrupt and the color of flow are given below table

Table 6.4 show flow of color from left to right

\begin{tabular}{|l|l|l|}
\hline 25 & 15 & 77 \\
\hline 42 & 26 & 23 \\
\hline 30 & 11 & 15 \\
\hline
\end{tabular}

5 Else if the color of way in right to left than the image is not corrupt and the color of flow are given below table

Table 6.5 show flow of color from right to left

\begin{tabular}{|l|l|l|}
\hline 77 & 15 & 25 \\
\hline 23 & 26 & 42 \\
\hline 15 & 11 & 30 \\
\hline
\end{tabular}

6 Else if the color of way top right to bottom left than the image is not corrupt the color of flow are given below table

Table 6.6 show flow of color from way top right to bottom left.

\begin{tabular}{|l|l|l|}
\hline 200 & 100 & 245 \\
\hline 10 & 210 & 11 \\
\hline 144 & 145 & 90 \\
\hline
\end{tabular}

7 Else if the color of way bottom left to top right than the image is not corrupt and the color of flow are given below table

Table 6.7 show flow of color from way bottom left to top right.

\begin{tabular}{|c|c|c|}
\hline 40 & 11 & 50 \\
\hline 78 & 12 & 86 \\
\hline 13 & 15 & 14 \\
\hline
\end{tabular}

8 Else if the color of way top left to bottom right than the image is not corrupt and the color of flow are given below table

Table 6.8 show flow of color from way top left to bottom right.

\begin{tabular}{|l|l|l|}
\hline 144 & 100 & 90 \\
\hline 11 & 210 & 10 \\
\hline 200 & 145 & 245 \\
\hline
\end{tabular}

9 Else if the color of way bottom right to top left than the image is not corrupt and the color of flow are given below table 
Table 6.9 show flow of color from way bottom right to top left.

\begin{tabular}{|l|l|l|}
\hline 90 & 145 & 144 \\
\hline 10 & 210 & 11 \\
\hline 245 & 100 & 200 \\
\hline
\end{tabular}

The all above table show error free pixel value and not change Other wise put center pixel value of following procedure:

9. The below table show the $\mathrm{W}_{3 \times 3}$ Matrix with error pixel find the above program

Table 6.10 show $X_{i, j}$ pixel is noisy

\begin{tabular}{|l|l|l|}
\hline 10 & 11 & 10 \\
\hline 10 & 12 & 11 \\
\hline 10 & 11 & 10 \\
\hline
\end{tabular}

The above filter solve the problem for the following formula

$$
\begin{aligned}
X_{i, j} & =(11+10+11+10+11+10+11+10) / 8 \\
& =10.5
\end{aligned}
$$

The value of 12 is replace by the $\mathrm{Xi}, \mathrm{j}$

\section{COMPARE PROPOSE FILTER WITH EXIST MDBUTMF HISTOGRAM ESTIMATION FILTER}

Quality of the filtering technique can be measured by comparing original image and the restored image statistics. Of course, peak signal to noise ratio (PSNR) would be the better option for assessing the restoration quality. PSNR is calculated through Mean Absolute Error (MAE). PSNR can be expressed as

$$
\begin{gathered}
P S N R=10 \times \log _{10}\left(\frac{255^{2}}{M S E}\right) \\
M S E=\frac{1}{N_{1} \times N_{2}} \sum_{i=1}^{N_{1}} \sum_{j=1}^{N_{2}}(x(i, j)-\hat{x}(i, j))^{2}
\end{gathered}
$$

Where, PSNR is in $\mathrm{dB}$. $\mathrm{X}$ is the original image with noise. $\mathrm{X}^{\wedge}$ is the de noised image. $\mathrm{M} \times \mathrm{N}$ represent the size of the image.

Calculate the PSNR (Peak signal to noise ratio) for the following formula

$$
\mathrm{PSNR}=10 \log _{10}\left(\frac{\operatorname{Square}(R)}{M S E}\right)
$$

Here firstly calculate the MSE for the following formula

$\mathrm{MSE}=\mathrm{SSE} / 2$

Where SSE is Sum of square Error

So calculate the SSE of the following number
Table 7.1 show the random value for calculating the SSE

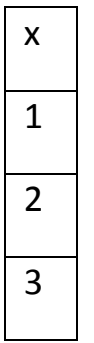

$$
\bar{X}_{\cdot 1}=\frac{\sum_{i=1}^{n} x_{i 1}}{n_{1}}
$$

$$
\vec{x}=2
$$

$\mathrm{SSE}=(1-2)^{2}+(2-2)^{2}+(3-2)^{2}$

$=1+0+1$

$=2$

MSE $=2 / 3$

$$
=0.6666667
$$

$$
\begin{aligned}
\text { PSNR } & =10 \log _{10} \frac{\text { square }(255)}{0.66667} \\
& =49.89
\end{aligned}
$$

\section{COMPARISON PSNR VALUES OF MDBUTMF HISTOGRAM ESTIMATION FILTER WITH LHM FILTER AT DIFFERENT NOISE DENSITIES}

Table 8.1 Shows Compare Both Filter Noise Density vs PSNR

\begin{tabular}{|c|c|c|}
\hline $\begin{array}{c}\text { Noise Density } \\
(\%)\end{array}$ & $\begin{array}{c}\text { MDBUTMF with } \\
\text { Histogram Estimation }\end{array}$ & Propose Filter \\
\hline 10 & 38.9 & 39.23 \\
\hline 30 & 32.65 & 32.79 \\
\hline 50 & 29.05 & 29.63 \\
\hline 70 & 26.29 & 26.78 \\
\hline 90 & 18.77 & 22.14 \\
\hline
\end{tabular}




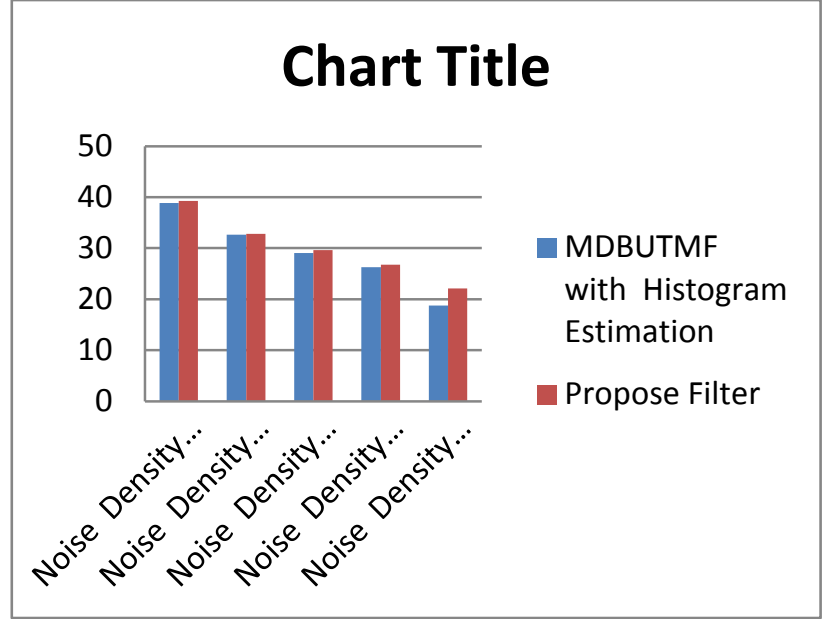

Figure 8.1:-compare Both Filter graph

\subsection{Comparison MSE Values of MDBUTMF Histogram Estimation Filter with LHM Filter at Different Noise Densities}

Table 8.2 Shows Compare Both Filter Noise Density vs MSE

\begin{tabular}{|c|c|c|}
\hline $\begin{array}{l}\text { Noise } \\
\text { Density } \quad(\%)\end{array}$ & $\begin{array}{l}\text { MDBUTMF with } \\
\text { Histogram Estimation }\end{array}$ & Propose Filter \\
\hline 10 & 8.56 & 7.76 \\
\hline 30 & 36.12 & 34.17 \\
\hline 50 & 80.54 & 70.80 \\
\hline 70 & 152.46 & 136.44 \\
\hline 90 & 420.68 & 396.66 \\
\hline
\end{tabular}

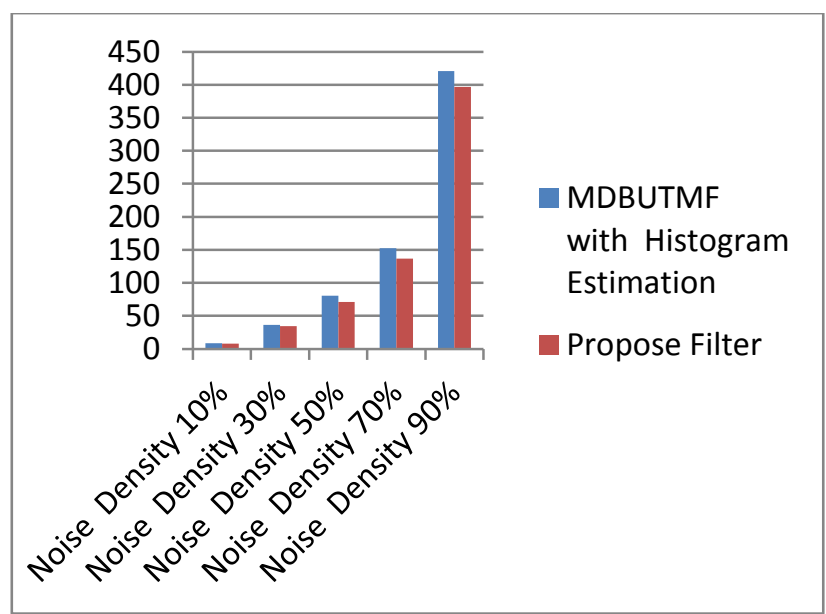

Figure 8.2 Compare Both Filter graph

\section{CONCLUSION}

In this paper we proposed an effective technique to improve the quality of image in LHM Filter. The LHM to restore the images which are corrupted with high density Noise.The above filter removes the salt and pepper noise with random noise. The proposed algorithm tries to identify noisy pixels and restore them properly and the quantitative comparison of proposed algorithm is also done with the existing noise removal algorithms. This algorithm is done in MATLAB 7.11 R2010b (32 bit). The performance of the algorithm has been tested on both color and grayscale images at varying noise densities. It is evident from the experimental results that proposed algorithm gives better performance both visibly and quantitatively. This work can be carried further for improvement of the quality of the output image

\section{FUTURE SCOPES}

In future, for the enhancement purpose, more images can be taken from the different application fields such as

\subsection{Image Processing in Satellite}

Today, many Satellite which is above our Earth. It is send us images of our Earth. So we free the image noise is guessing about the upcoming season. How will the coming season. Where will the rain. Where will the fog. Where will foot mist. Where clear weather.

\subsection{Image-processing system uses in Education}

the image capturing from planet and analysis to here the propose filter is very useful to space image analysis if image transmit from source to destination the quality of image is not similar to source image then above filter is used to remove the noise to destination image so as above filter is very useful in image transmission field.

\subsection{Image Processing and its Military Applications}

Image processing - a moving horizon! Walking towards a horizon is open ended. The horizon never gets any closer to you, but continually recedes from you. Thus it has been with the growth of image processing, as a technical discipline. Constant progress is being made - but the potential is far from exhausted. In the early years of image processing the concern was of basic phenomena, for example, making models for image data compression, image restoration and image enhancement. Currently there is a great interest in moving beyond physical phenomena and into the realms that are wrapped with psychology, perception and cognition. The research in this branch of image processing is often called as 'Image Understanding'.

The ability to place earth oriented sensors into orbit, because of its large economic Potential has received considerable research emphasis, and many operational systems have been evolved. Land remote sensing has been evolving since 1960s and its various applications in urban land utilization, forestry and food commodity production forecasting has been producing remarkable results. An off shoot of remote sensing by satellites has been the use of this data for military purposes.

\section{REFERENCES}

[1] Prateek Bansal, Himanshu Yadav,,Ramesh Kumar Sunkaria "Impulse Noise Removal Using MDBUTMF with Histogram Estimation" 2015 IEEE

[2] Aref Shams-Baboli, Ali Akbar Shams-Baboli," A Modified Nonlinear Filtering Technique for Removal of High Density Salt and Pepper Noise" November 18 19, 2015 IEEE 
[3] Er.Ravi Garg and Er. Abhijeet Kumar, "Comparison of Various Noise Removals Using Bayesian Framework", International Journal of Modern Engineering Research (IJMER) , Jan-Feb 2012, Vol.2, Issue.1, 265-270.

[4] Mohammed Ghouse, Dr. M. Siddappa, "Adaptive Techniques Based High Impulsive Noise Detection and Reduction of Digital Image", Journal of Theoretical and Applied Information Technology, Vol.24, No.1.

[5] Shyam Lal, Sanjeev Kumar and Mahesh Chandra "Removal of High Density Salt \& Pepper Noise through Super Mean Filter for Natural Images" IJCSI International Journal of Computer Science Issues, Vol. 9, Issue 3, No 3, May 2012.

[6] Geeta Hanji, M.V. Latte "A novel adaptive tolerance filter for random valued impulse noise suppression in digital grey and color images" Journal of Advanced Computer Science \& Technology, 4 (1) (2015) 43-52

[7] Saleh Saraireh, Yazeed Al-Sbou, Ja'afer Al-Saraireh, Othman Alsmadi "Image Encryption Scheme Based on Filter Bank and Lifting" Int. J. Communications, Network and System Sciences, 2014, 7, 43-52 Published Online January 2014.

[8] Hossein Hosseini and Farokh Marvasti "Fast restoration of natural images corrupted by high-density impulse noise"
Hosseini and Marvasti EURASIP Journal on Image and Video Processing 2013, 2013:15

[9] Anjali Garg “An Improved Switching Median Filter Based on Local Outlier Factor" International Journal of Engineering Research and General Science Volume 3, Issue 2, March-April, 2015

[10] S. Athi Narayanan, G. Arumugam, Prof. Kamal Bijlani" Trimmed Median Filters for Salt and Pepper Noise Removal" Volume 2, Issue 1, January - February 2013

[11] Anup Vibhute, S K Bodhe "Applications of Image Processing in Agriculture: A Survey"International Journal of Computer Applications (0975 - 8887) Volume 52 - No. 2 , August 2012

[12] B.Srikanth, G.Padmaja , Dr. Syed Khasim , Dr. P.V.S.Lakshmi, A.Haritha "Secured Bank Authentication using Image Processing and Visual Cryptography " Vol. 5 (2) , 2014, 2432-2437

[13] "An Investigation of an Image Processing Application for Measurement of a Quantity Estimate of Euro Form Installed in Construction Sites "16-18 Dec. 2010

[14] V.V.D. Shah "Image Processing and its Military Applications" Military College of Telecommunication Engineering, Mhow4.53 4412015 IEEE 